\title{
Immunodiagnosis of Neurocysticercosis: Ways to Focus on the Challenge
}

\author{
M. Esquivel-Velázquez, P. Ostoa-Saloma, J. Morales-Montor, \\ R. Hernández-Bello, and C. Larralde
}

Departamento de Immunología, Instituto de Investigaciones Biomédicas, Universidad Nacional Autónoma de México (UNAM), A.P. 70228, Ciudad Universitaria, C.P. 04510 México City, DF, Mexico

Correspondence should be addressed to C. Larralde, larralde@servidor.unam.mx

Received 1 June 2011; Revised 22 August 2011; Accepted 23 August 2011

Academic Editor: Luis I. Terrazas

Copyright (๑) 2011 M. Esquivel-Velázquez et al. This is an open access article distributed under the Creative Commons Attribution License, which permits unrestricted use, distribution, and reproduction in any medium, provided the original work is properly cited.

Neurocysticercosis (NCC) is a disease of the central nervous system that is considered a public health problem in endemic areas. The definitive diagnosis of this disease is made using a combination of tools that include imaging of the brain and immunodiagnostic tests, but the facilities for performing them are usually not available in endemic areas. The immunodiagnosis of NCC is a useful tool that can provide important information on whether a patient is infected or not, but it presents many drawbacks as not all infected patients can be detected. These tests rely on purified or semipurified antigens that are sometimes difficult to prepare. Recent efforts have focused on the production of recombinant or synthetic antigens for the immunodiagnosis of NCC and interesting studies propose the use of new elements as nanobodies for diagnostic purposes. However, an immunodiagnostic test that can be considered as "gold standard" has not been developed so far. The complex nature of cysticercotic disease and the simplicity of common immunological assumptions involved explain the low scores and reproducibility of immunotests in the diagnosis of NCC. Here, the most important efforts for developing an immunodiagnostic test of NCC are listed and discussed. A more punctilious strategy based on the design of panels of confirmed positive and negative samples, the use of blind tests, and a worldwide effort is proposed in order to develop an immunodiagnostic test that can provide comparable results. The identification of a set of specific and representative antigens of T. solium and a thorough compilation of the many forms of antibody response of humans to the many forms of T. solium disease are also stressed as necessary.

\section{Introduction}

Neurocysticercosis (NCC) is a disease caused by the metacestode or larval form of the tapeworm Taenia solium when it lodges in the central nervous system (CNS) and is endemic of the Andean area of South America, Brazil, Central America and Mexico; China, the Indian subcontinent, and SouthEast Asia; sub-Saharan Africa [1-3]. It is considered a public health problem as it is the main cause of late-onset epilepsy [4] and it is also the most important parasitic disease of the nervous system $[3,5,6]$. Cysticerci may also locate elsewhere in skeletal muscles, heart, eyes, diaphragm, tongue, and subcutaneous tissues, causing a condition simply referred to as cysticercosis.
NCC is a disease difficult to diagnose based on the clinical picture as it presents a variety of nonspecific symptoms and in $50 \%$ of the cases none [7]. The symptoms differ according to the location of the cysts in the brain (parenchymal or ventricular) and the number and the state of the parasites (vesicular, degenerating, or calcified) [5, 8-12]. The severe forms of NCC seriously impair the patients' health and may lead to death. Medical diagnosis of NCC is impossible on clinical data alone. The definitive diagnose is made using a combination of methods including images of the cysts in the brain (by computed tomography or magnetic resonance imaging) and immunological methods (detection of specific antibodies or antigens). As this is a disease frequently associated to poverty $[3,13,14]$, the availability and high 
costs of neuroimages or sophisticated immunological assays in endemic areas limit the diagnostic capacity [15]. An effective immunodiagnosis of NCC would be the most practical way to facilitate medical diagnosis for millions of poor people in endemic countries and it would also supply sero-epidemiological studies with a low-cost indicator of prevalence of infection. In addition, a positive immune test would raise the clinical suspicion of early nonsymptomatic NCC which, if confirmed, would allow offering early treatment. Interesting advances in immunodiagnostic assays for NCC have been made during the past few years, involving the use of synthetic or recombinant antigens [16-29] and some efforts have been done to detect specific antigens or antibodies in noninvasive ways for the patient [30-33]. Most reports initially claim very high specificity/sensitivity scores, sometimes even as high as 100/100\%. Enthusiasm soon calms as the methods are applied by different laboratories, in larger numbers of cases and in various epidemiological scenarios of the disease [31, 34-37].

Immunodiagnosis of NCC can be done by two ways: by identifying antibodies against cysticercal antigens, or by identifying parasite's antigens directly. Here is a review of the recent studies made in the area of immunodiagnosis of NCC and the methods used in each case are discussed.

\section{Biological Factors Involved in Neurocysticercosis}

Host factors as age, gender, or race are involved in the severity of NCC (Figure 1). Age has an effect upon the number of cysticercal lesions and on the state of the cysticerci in the brain (vesicular, colloidal): while vesicular cysticerci increase with aging, colloidal cysticerci diminish without representing an increment on severity of NCC [38]. Sexual dimorphism has been reported in many parasitic infections as malaria, schistosomiasis, tripanosomiasis, toxoplasmosis, and cysticercosis [39]. The effect of sex hormones upon the immune system is evident, for example, in T. solium cysticercosis, the prevalence of naturally infected pigs almost doubles in castrated or pregnant pigs [40]. In general, females generate more robust humoral and cell-mediated immune responses than males, but males present a stronger inflammatory response to infectious organisms [41]. In NCC, females present increased levels of IL5/Il6/IL10 in cerebrospinal fluid (CSF) [42] as well as a higher leukocyte counts than men [38] and more frequently present severe NCC [43] which may have a relation with the location of the cysts in the brain and with higher inflammatory profiles in female [44]. Furthermore, females present higher immunoglobulin levels than men to different antigenic challenges [45] and in seroepidemiological surveys females show the highest anticysticerci response [46].

The genetic differences between different populations also play a role in infectious diseases [47-49], especially on terms of susceptibility to disease as in the case of malaria [50]. In NCC, some antigens from the HLA complex have been involved in the relative risk for developing parenchymal NCC [51], but studies of this type are scarce. A study identi- fying genes responsible for the pleomorphic presentation of the disease has not been done, but the effect of the genetic background upon the development of this particular disease is clear between subjects from different endemic areas, as is the case of India in which single cysticercotic granuloma (SCG) is the most frequent presentation of NCC while in Latin America it is not $[35,52]$.

Furthermore, genetic variability of the parasite itself has been described. Genetic variability of cysticerci has been found at different levels, from the global level (which identified two genotypes: Asia and Africa/Latin America) [53-57]) to the regional and the community level [58].

With all these factors involved, it is hard to find a single immunodiagnostic test that can detect all true NCC cases and that can work worldwide.

\section{Problems, Advances, and Perspectives in the Immunodiagnosis of Neurocysticercosis}

Historically, tests developed to diagnose NCC either by detecting specific antibodies or antigens have shown that not all cases could be detected (false negatives) and many other cases are detected as positive (false positives) when they are not. The first case is related to the number, state, and location of the cysticerci in the brain and involves patients with degenerating, dead, or single cysticerci. The second case involves patients with parasitic diseases closely related to cysticercosis (as echinocococcis or hymenolepiasis), patients exposed to the parasite but that did not become infected, patients with cysticercosis outside the central nervous system and patients who once were infected but resolved the infection without consequences. Additionally, almost half of NCC cases are asymptomatic [12] and the symptomatic cases present a variety of unspecific symptoms as chronic epilepsy and headaches as the most common [8].

Immunodiagnostic tests for NCC initially claim to have very good sensitivity/specificity scores, but as they are being tested by other groups, in larger number of cases and in areas with different degrees of endemicity the scores are lower [31, 37, 52]. Also the scores lower because many immunodiagnostic tests rely on purified or semipurified antigens and the procedure of purification is complex and frequently require technical expertise. This question, coupled with differences in the selection of the NCC patients and the control groups, provokes large variations within and between tests and low reproducibility between laboratories. The need to find new antigens for immunodiagnosis of NCC which can improve the diagnostic capacity of actual tests persists. These new antigens have to be tested by various laboratories to prove that the sensitivity/specificity maintains between tests putting special attention on the selection of NCC patients and controls so the results can be compared. Some efforts have been done in recent years to try to make a more uniform immunodiagnostic test $[20,24]$ and to make comparable the results from different tests between laboratories [16, 26, 59, $60]$. 


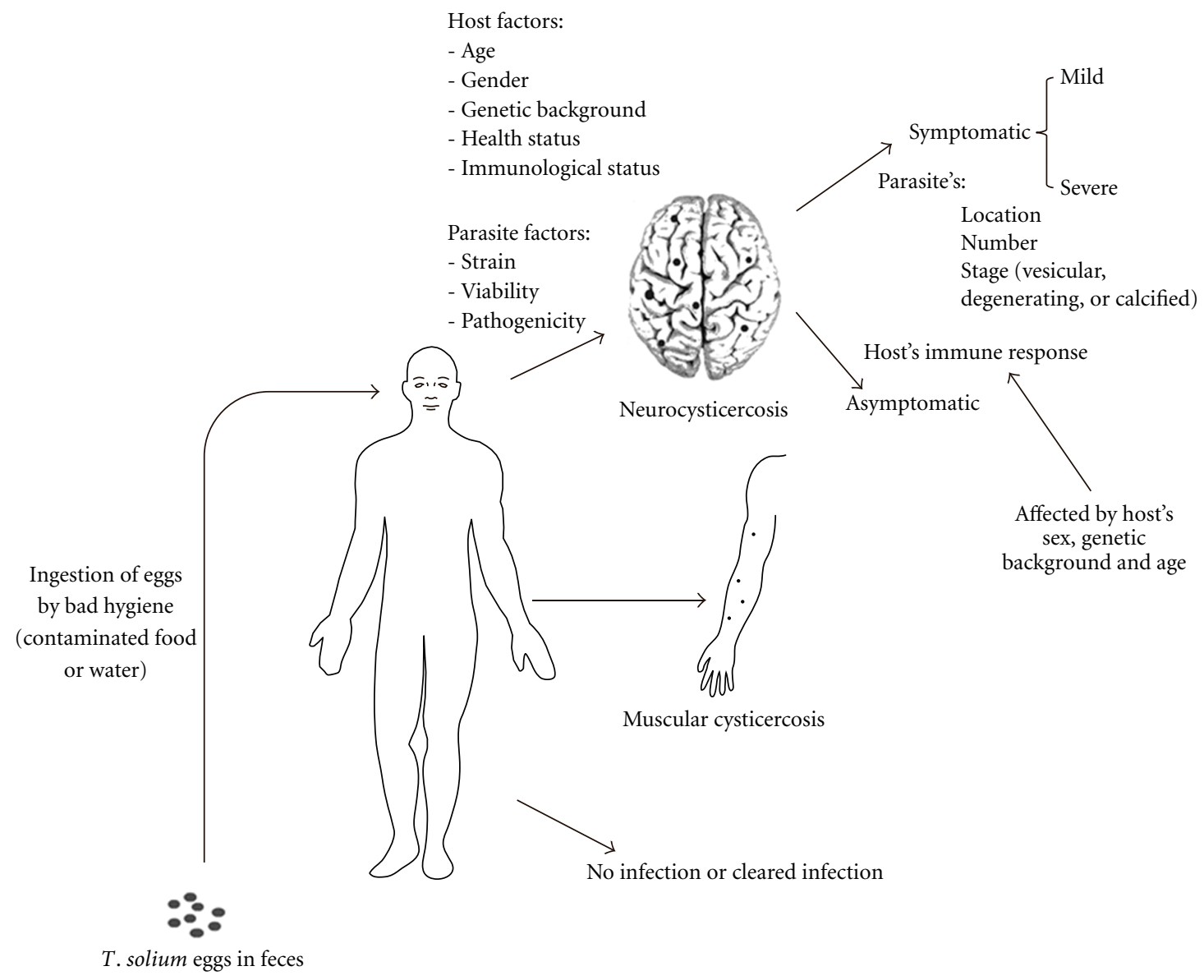

FIGURE 1: Factors involved in NCC. The development of NCC depends on many factors from either the host or the parasite. The factors affecting the immune response of the host are particularly important for the immunodiagnosis of NCC as they may affect the results between individuals.

\section{Detection of Antigens or Antibodies for the Immunodiagnosis of NCC}

Immunodiagnostic tests can be divided in two major groups: the ones that use an antigenic mixture or single antigens to try to find antibodies against them; and the ones that use specific antibodies to find specific antigens in the samples.

Looking for antibodies against cysticercal antigens can be done using a variety of samples from the human body: serum, CSF, urine, saliva, and so forth, but a major disadvantage of this approach is that false positives can result as antibodies do not necessarily indicate an active infection with viable metacestodes but a resolved one or exposure to the parasite [61]. Another disadvantage is that cross-reactivity may occur with other parasitic diseases, most commonly Echinococcus granulosus [25, 62-67], although cross-reactivity has also been reported with other diseases as hymenolepiasis, fasciolasis [62], toxoplasmosis [67, 68], malaria [67], amoebiasis [67], syphilis [68], hepatitis B [68], toxocariasis [62], cerebral tuberculosis [37, 67], mononucleosis [68], among others $[25,37,65]$, which may be due to the selection of bad characterized samples and not necessarily to the fact that patients are infected with something else than T. solium. However, the search for antibodies in samples has the advantage that mixtures of antigens can be used (as parasite extracts or semipurified antigens) while the search for antigens needs to have specific antibodies against the desired antigens but can indicate an active infection $[15,69]$. When looking for antibodies against parasite antigens, the most frequently used samples are serum or CSF and the most common immunoglobulin is IgG as it is the predominant antibody detected in NCC, although $\operatorname{IgA}, \operatorname{IgE}$, and $\operatorname{IgM}$ can also be detected but have little value in diagnosis [13, 70], though they can be used for follow-up, as NCC patients show undetectable levels of IgG4, IgM, and IgA antibodies in saliva after drug treatment, while antibodies in serum persist longer regardless of the subtype [33].

\section{Antigens Used for Inmunodiagnosis of NC}

Multiple antigens have been used for the immunodiagnosis of cysticercosis, among them are low molecular mass (LMM) antigens [31], excretory/secretory (ES) [30, 31, 71-74], crude soluble extract (CSE) [31, 68], total saline extract [59], antigen B [75, 76], lentil lectin glycoproteins (LLGPs) [52], 
vesicular fluid (VF) $[68,77]$, membrane and scolex extracts [68], somatic antigens [74], recombinant proteins [16-18, $20,21,25-28]$, and synthetic peptides [19, 24, 29]. The source of these antigens has been commonly Taenia solium (the parasite responsible for NCC) but related species as Taenia crassiceps $[5,24,68,77-79]$, Taenia saginata $[59,80]$, or Taenia taeniformis [81] have also been used as antigen sources; and among the multiple methods used to date for the immunodiagnosis of NCC complement fixation, agglutination, radioimmunoassays, ELISA and Western Blot (WB) can be counted [34, 77, 80, 82, 83]. Some of these methods are too old, but have the advantage of being cheap, so they could be used with new antigens and yield different results.

Most commonly for the immunodiagnosis of NCC, serum or CSF samples are used with some advantages and disadvantages for each one. For instance, it is proposed that the detection of antigens or antibodies in CSF is better than in serum because there is a release of parasite antigens directly to it or local production of antibodies, but it is more difficult and dangerous to obtain than serum and requires special facilities. However, WB with LLGPs (LLGP-WB, considered by many to be the most reliable method for serologically detecting NCC) both samples have no significant differences in performance, although antigen detection by ELISA is better in CSF than in serum, but this test is less sensitive than LLGP-WB [69].

LLGP-WB [84] has a sensitivity of $>90 \%$ and a specificity of $100 \%$ [2]. This assay involves the separation of 7 glycoprotein antigens $(50,42-39,24,21,18,14$, and $13 \mathrm{kDa})$ by SDS-PAGE and its recognition in an immunoblot by serum of CSF antibodies. However, in recent studies mainly in Indian patients where almost two thirds of the NCC patients have an SCG $[35,52]$, LLGPs have shown to be less sensitive than for multiple cysticerci. In these cases, sensitivity has been reported to range between 50 and $80 \%$ and specificity between 94 and 100\% [22, 35, 85, 86]. Also this test has been shown to be less sensitive in children than in adults and the pattern of protein recognition also differed in these age groups (children tend to recognize the higher molecular mass proteins while adults tend to recognize LMM with serum or CSF antibodies) [87] probably due to the time of infection and the antigenic stimuli. The most common bands identified by serum antibodies are, along with the 18 and $14 \mathrm{kDa}$, the $29 \mathrm{kDa}$ for SCG and the $31 \mathrm{kDa}$ for multiple lesions [35]. In Latin American NCC patients, where multiple lesions are more common than SCG, the most common recognized bands were 39$42 \mathrm{kDa}$ and $21 \mathrm{kDa}$ [60]. So there seems to be differences not only in the amount of antibodies produced between SCG and multiple lesions [88, 89], but also in the antigens recognized by these antibodies. To try to improve the diagnosis of SCG patients, LLGPs have been unfolded and reduced trying unmask epitopes that may detect antibodies in these SGC patients and resulted that the unfolding of the diagnostic proteins with urea exhibited the maximum antibody binding and in this conformation $46 \%$ of the patients with SGC that were serologically negative became positive [35].
Problems like this one, or the difficulty to differentiate between NCC and cysticercosis elsewhere have lead to the search of new antigens and methods to immunodiagnose NCC. Antigenic extracts from Taenia solium cysticerci have been reevaluated $[31,68,90]$, as well as antigens from other related species as Taenia taeniformis [81], Taenia saginata $[59,80]$ or Taenia crassiceps $[5,24,68,77-79]$, which have been useful for identifying new protein candidates for the immunodiagnosis of NCC and also have shown drawbacks as the propensity to cross-reactions which has lead to tests with low specificity. However, the use of related species as the ones mentioned above provide an alternative source of parasitic antigens as there are difficulties to obtain Taenia solium cysticerci from a natural infected source, even in areas of high endemicity $[78,91]$. However, the use of parasitic extracts present many drawbacks that need to be considered if intended for immunodiagnosis as the variation between isolates and differences in the methods of extraction by different laboratories, which leads to poor reproducibility and invalid comparisons between results.

\section{Advances in the Methods for Inmunodiagnosis of NC}

New methods for the immunodiagnostic of NCC have also been developed involving the reevaluation of common methods with new antigens (e.g., ELISA or WB), or the use of different samples as urine or saliva [30, 31, 33], as they have the advantage that they are very simple to obtain and do not cause any harm to the patient. They are also convenient to assay in endemic areas as facilities to obtain CSF or serum samples are not frequently present [92]. However with these samples not very good results have been obtained in terms of sensitivity or specificity, especially with patients with SCG, and the methods need to be validated with well-defined positive and negative NCC samples and with samples from other parasitic diseases. In the case of urine, sensitivity has been reported to be of $92 \%$ which decreased to $62.5 \%$ for SCG patients when antigens were detected with monoclonal antibody-ELISA [32]. Results were not very good for antibody detection either by ELISA or LLGP-WB in urine, with reported sensitivities ranging from 44 to $76 \%$ and specificities from 33 to $66 \%$, despite the antigen used (ES, LMM, or CSE) [30, 31]. Saliva has been tested for the detection of antibodies against CSE or antigen B (composed by 2 immunologically identical polypeptides prepared by collagen-binding method $[75,76]$ ) with no much better results for any of the immunoglobulins tested (IgG, IgG1, IgG4, IgM, and IgA), although it was shown that IgG4 in saliva could be a useful tool for patients' follow-up after treatment [33]. Nonetheless, these and other studies lack the appropriate controls (well-defined positive and negative samples, and well-defined samples of patients with other infections to assess cross-reactions). Another method that has emerged for the diagnosis of NCC, although it is not immunologically very interesting, is the amplification by PCR of T. solium DNA present in the CSF of NCC patients, with a high reported sensitivity of $96.7 \%$ [93], though 
control samples from patients with other parasitic infections were not included. Other methods for the immunodiagnosis of NCC have also been tried in an attempt to substitute the LLGP-WB assay as its performance is expensive and requires technical expertise. Among these methods is the dot blot which is easier to perform and has shown to have sensibility and specificity similar to ELISA [64, 67, 90, 94].

In addition, there have been efforts to try to correlate the result in an immunodiagnostic test with the location and state of the cysticerci in the brain. An example is the detection of the antigen HP10 in CSF, which correlates with the location of the cyticerci in the brain: when located in the subarachnoideal space or the ventricles, HP10 could be detected, but when located in the parenchyma HP10 could not be detected; and when cysts were damaged, HP10 levels were reduced significantly [15]. Also high antigen levels in CSF suggest the presence of subarachnoid NCC [69]. Many studies have reported tests that can differentiate live from dead cysticerci by the detection of excretory/secretory (ES) antigens in CSF or serum [71-74], or by the detection of antibodies against a $10 \mathrm{kDa}$ protein from the vesicular fluid of Taenia solium $[17,25]$. These studies support the idea that infected hosts produce antibodies of different specificities as the cysticercus develops, degenerates, and dies because the antigens released by the parasite in each state are different [72].

Many of the most recent efforts in the field of immunodiagnosis of NCC have centered in the production of recombinant proteins or synthetic peptides that could provide a reliable source of antigens without depending on obtaining cysts from naturally infected hosts. The advantage of these antigens is that they would make comparable results between and within laboratories as there is no need to purify cysticercal antigens. However, synthesized or recombinant proteins do not have the same glycosilation pattern as those obtained directly from the parasite (recombinant proteins) or do not have any glycosilation at all (chemically synthesized proteins). This often affects the sensibility of the produced protein, which is frequently assessed with sera that were positive to the native protein, but rarely with different patients' sera to assess if the produced protein could detect more cases than the native one $[16,19-21]$. A sensitivity of $95 \%$ means that the produced protein can detect $95 \%$ of the cases that the native protein detected, though that does not represent a real improvement in immunodiagnosis of NCC unless it can also detect cases that the native protein could not.

The principal protein targets to produce or synthesize are the components of the LLGP-WB that are recognized by NCC patients, and several of them have been already synthesized and tested preliminarily. For instance, from the members of the $8 \mathrm{kDa}$ family, Ts18var1 has been produced in insect cells [16] as well as TsRs1, Ts18var1, and Ts18 Var3 [20]; the 14 and $18 \mathrm{kDa}$ proteins produced by recombination [27]; Ts14, Ts18var1, TSRS1, and TSRS2var1 by chemical synthesis [29], and full-length Ts18 and Ts14 by chemical ligation [19], $\mathrm{Ag} 1 \mathrm{~V} 1 / \mathrm{Ag} 2$ by recombination [26] as well as Ts8B1, Ts8B2, Ts8B3 [18], Ts14 [27] and a $10 \mathrm{kDa}$ protein $[17,25]$; GP50, which is not a member of the
$8 \mathrm{kDa}$ family but it is part of the LLGPs, was produced by recombination in bacteria and in a baculovirus expression system $[16,21]$. Other proteins outside those from LLGPs that have also been produced or synthesized include T24 (integral membrane protein that does not bind to lentil lectin) produced in a droshophila cell line [22]; HP6-Tsag (oncospheral adhesion protein of Taenia saginata) in bacteria and baculovirus systems with similar specificities between the systems (93-95\%), but higher sensitivity for the inactive cases by the baculovirus protein (48-64\%) [95]; peptide NC-1 selected by phage-display [23]; peptides KETc12, 410, and 413 synthesized from a cDNA library of T. crassiceps [24], and recombinant TS24 and Es33 [28]. The methods of production are varied, as well as the results and the ways to evaluate the produced protein, some giving very good sensitivities but in other cases, the native protein is much better than the produced one.

Finally a very interesting approach for the diagnosis of cysticercosis in pigs has been developed. This approach involves the production of nanobodies (camelid-derived single-domain antibody fragments) by recombination after immunizing dromedaries with cisticercal antigens its evaluation for serodiagnosing cysticercosis in pigs. The selected nanobodies had the advantage that did not cross-react with other closely-related parasitic diseases as Taenia hydatigena, Taenia saginata, Taenia crassiceps, or Trichinella spiralis, although cross-reactivity with other parasites as Echinococcus granulosus was not assessed. Nanobodies are heavy-chainonly antibodies that recognize antigens as firmly as normal antibodies do but are about one tenth their size [96]. This characteristic allows them to often recognize epitopes that are not readily accessible to conventional antibodies [97]. Nanobodies have been used also in the diagnosis of trypanosomiasis [98] or malaria [99], but mostly there are being directed to treat more efficiently autoimmune diseases or cancer than commercial conventional antibody therapies [100]. Nanobodies have beneficial production and stability properties [97] which, along with their antigenrecognition characteristics, make them a promising tool for the diagnosis and treatment of many diseases in which NCC may be included, and this approach should be translated to the NCC field as it may provide an assay with higher sensitivity, especially for diagnosing SCG patients which are often negative in conventional tests, by recognizing epitopes that are not recognized by conventional antibodies.

Despite all these efforts and alternatives, a definitive immunodiagnostic test for NCC has not been achieved. The need to account with a more reproducible and sensitive immunodiagnostic test than the actual ones remains, and some studies have focused on this issue by synthesizing antigens from LLGPs used in WB and other antigens, but still there is the need to find new antigens that can detect those cases that are negative in LLGP-WB like SCG cases. Different protein expression systems have been tried to overcome the problem of requiring a natural source of antigens and the difficulties of purifying cysticercal antigens, but the produced proteins lack the natural glycosilation pattern and show diminished sensitivity than the native proteins. New approaches to diagnose NCC should be explored, as 
the use of nanobodies that could result very interesting in detecting difficult cases. Developing an immunodiagnostic test that could detect $100 \%$ of the true NCC cases and exclude $100 \%$ of the true negative cases has been difficult, especially considering biological factors that are involved and the many forms of cysticercosis as differences in the genetics of the host and of the parasite, and there is still much to do to improve the current tests. The biological factors, especially those related to genetic differences of the host or the parasite, that affect NCC may explain why a particular immunodiagnostic test first reports very high sensitivity and specificity scores that then lower as it is being applied in different regions and by different laboratories; and these factors could also make impossible the development of a single immunodiagnostic test, but local tests that can detect cases in a certain endemic area can be developed.

Special attention should be paid in the cases selected to evaluate new tests so results can be compared with other tests, especially in the cases selected as controls (healthy individuals and with other parasitic diseases) as these are responsible for the specificity reported and an adequate selection can assure that results can be compared between tests. Also, attention should be put in including different subsets of NCC clinical types, as these differences affect the amount of antibodies or antigens that can be detected. Extraparenchymal forms of NCC are associated with higher circulating antigen levels and more reactive antibody bands in LLGP-WB than intraparenchymal forms [69, 101-104]. These differences can affect the estimation of the performance of newly developed tests, so attention should be put in the number of cases of each sub-type that are included.

A plausible approach to identify antigens that can be useful for the serodiagnosis of NCC is the separation in 2 dimensions of cysticercal antigens and their recognition by hosts' immunoglobulins. This method can give us useful information about the differences in recognition of the parasite's antigens by different hosts (immunological diversity) and about the antigens that are recognized by many or all infected hosts (if there are any) to select antigens for use in an immunodiagnostic test to detect true positive cases.

Finally, the following proposals for improving the actual immunodiagnostic tests are made.

6.1. Proposals for Improvement. (1) T. solium disease is present in many countries around the world and many research groups are working to develop an immunodiagnostic test that can detect all NCC individuals although so far that goal has not been accomplished. Cooperation is necessary to concert a worldwide effort to carefully design a research plan concordant with the complexities of T. solium disease, and to develop and test in the short term with a minimal number of options from which to select the most proficient immunodiagnosis of NCC the possibility to be put to immediate production and general use while further research for improvement continues.

(2) Clearing the problem of antigen cross-reactivity and species representation is necessary to succeed in developing an immunodiagnostic test for NCC. Purification of antigen(s) or epitopes critically certified to be exclusive of $T$. solium and present in all members of a representative sample of parasite specimens of an endemic site is mandatory. Some likely candidates have been proposed [105], although further research is necessary to determine if they fulfill the conditions mentioned above. A way of avoiding the high costs and demanding technical skills involved in the purification of natural antigens is the use of those present in phage display peptide libraries [23] or the production of recombinant or chemically synthesized antigens [106]. Antigens present in only $T$. solium but not in other Taenia species would constitute the candidate antigen preparation (CAP).

(3) It is also necessary to study and characterize the presumed wide spectrum of humans' antibody production in T. solium disease in order to calibrate the candidate antigen preparation that would include all infected individuals. Western Blots using CAP in reaction with representative samples of all subsets of infected individuals (regardless of whether the parasite had established or not) if possible, or at least of confirmed cysticercosis and NCC samples, would provide the images necessary to construct all immunological profiles of the infected individuals. Computer-assisted image analysis of $\mathrm{WB}$ and cluster analysis could address this problem. The set of CAP that reacts with all or most infected individuals in which the parasite was established would constitute the definitive antigen preparation (DAP).

(4) Rather than attempting to develop ways to distinguish each of the different subsets of NCC disease, efforts in immunodiagnosis could focus on improving diagnosis of NCC (to include all NCC and NCC + cysticercosis samples and exclude cysticercosis, taeniosis, and infected but not established samples), while for the prevalence of T. solium disease, in whatever its form, it should only clearly distinguish members of the infected (established or not) from the not infected.

Three are the classes of $T$. solium disease that matter the most and perhaps require different strategies: the contact case, the NCC case (whether it is only NCC or NCC + cysticercosis elsewhere), and the tapeworm carrier. For this purpose, it is indispensable to construct representative and certified negative and positive control panels of the samples CSF, serum, and feces from each geographic area upon their reaction with DAP. Certification of the members of cysticercosis elsewhere and of the noninfected individuals is complicated by its need of whole-body scans in search of cysticerci located elsewhere of CNS. Additional negative control samples from a culturally and historically certified community or geographic area without $T$. solium disease and low in infectious diseases in general would be useful to establish the cut-off values for immunotesting with DAP.

(5) Once the problem of antigen specificity and representation is solved, there should be no major problem to Immunodiagnose NCC in the CSF of a symptomatic neurological patient nor of an intestinal tapeworm in the feces, preferably by antigen detection (this is to distinguish cysticercosis located elsewhere and live from dead cysticerci in the CNS because antibodies could persist after the death of the parasite for unknown periods of time). 
(6) However there would remain serious problems to tackle for serology, the most accessible sample useful for the detection of early nonsymptomatic NCC cases in the general population and for epidemiological studies of $T$. solium disease prevalence. The major problem for serology in unambiguously detecting asymptomatic NCC cases is the potential location elsewhere of the parasite (cysticercosis elsewhere or taeniosis) that produces false-positive results or the low reactivity of patients with few live cysticerci or with dead cysticerci (NCC or elsewhere) that produces falsenegative results. Adding to positive serology a marker of CNS damage $[107,108]$ as a sign of CNS involvement could help in discriminating NCC from other forms of T. solium disease.

The development of an effective and definitive immunodiagnostic test for NCC is possible, but a series of considerations and evaluations need to be addressed first as stated above, and a worldwide effort is required to develop a test that could be effective everywhere. Nonetheless, the effort is necessary and the result would be very useful to help eradicate this disease.

\section{Acknowledgments}

Financial support was provided by Grant no. IN204311-3 (P. Ostoa-Saloma) from Programa de Apoyo a Proyectos de Investigación e Innovación Tecnológica (PAPITT), Dirección General de Asuntos del Personal Académico, Universidad Nacional Autónoma de México. M. Esquivel-Velázquez is recipient of a doctoral scholarship from CONACYT (207061) for her PhD studies in the "Programa de Doctorado en Ciencias Biomédicas (PDCB)" at UNAM.

\section{References}

[1] H. H. García, A. E. Gonzalez, C. A. W. Evans, and R. H. Gilman, "Taenia solium cysticercosis," The Lancet, vol. 362, no. 9383 , pp. 547-556, 2003.

[2] A. C. White, "Neurocysticercosis: a major cause of neurological disease worldwide," Clinical Infectious Diseases, vol. 24, no. 2, pp. 101-115, 1997.

[3] World Health Organization (WHO), "Control of neurocysticercosis," in Proceedings of the 55th World Health Assembly, April 2002, Provisional Agenda Item 13.18.

[4] B. O. Colli, N. Martelli, J. A. A. Júnior et al., "Cysticercosis of the central nervous system. I. Surgical treatment of cerebral cysticercosis: a 23 years experience in the Hospital das Clínicas of Ribeirão Preto Medical School," Arquivos de Neuro-Psiquiatria, vol. 52, no. 2, pp. 166-186, 1994.

[5] R. H. S. Peralta, A. J. Vaz, A. Pardini et al., "Evaluation of an antigen from Taenia crassiceps cysticercus for the serodiagnosis of neurocysticercosis," Acta Tropica, vol. 83, no. 2, pp. 159-168, 2002.

[6] V. Rajshekhar, G. Chacko, R. P. Haran, M. J. Chandy, and S. M. Chandi, "Clinicoradiological and pathological correlations in patients with solitary cysticercus granuloma and epilepsy: focus on presence of the parasite and oedema formation," Journal of Neurology Neurosurgery and Psychiatry, vol. 59, no. 3, pp. 284-286, 1995.

[7] A. Fleury, J. Morales, R. J. Bobes et al., "An epidemiological study of familial neurocysticercosis in an endemic Mexican community," Transactions of the Royal Society of Tropical Medicine and Hygiene, vol. 100, no. 6, pp. 551-558, 2006.

[8] A. Fleury, M. Hernández, G. Fragoso, R. M. E. Parkhouse, L. J. S. Harrison, and E. Sciutto, "Detection of secreted cysticercal antigen: a useful tool in the diagnosis of inflammatory neurocysticercosis," Transactions of the Royal Society of Tropical Medicine and Hygiene, vol. 97, no. 5, pp. 542-546, 2003.

[9] H. H. Garcia and O. H. Del Brutto, "Neurocysticercosis: updated concepts about an old disease," The Lancet Neurology, vol. 4, no. 10, pp. 653-661, 2005.

[10] P. Salgado, R. Rojas, and J. Sotelo, "Cysticercosis: clinical classification based on imaging studies," Archives of Internal Medicine, vol. 157, no. 17, pp. 1991-1997, 1997.

[11] E. Sciutto, G. Fragoso, A. Fleury et al., "Taenia solium disease in humans and pigs: an ancient parasitosis disease rooted in developing countries and emerging as a major health problem of global dimensions," Microbes and Infection, vol. 2, no. 15, pp. 1875-1890, 2000.

[12] J. Sotelo and A. Flisser, "Neurocysticercosis. Practical treatment guidelines," CNS Drugs, vol. 7, no. 1, pp. 17-25, 1997.

[13] A. Carpio, "Diagnostic criteria for human cysticercosis," Journal of the Neurological Sciences, vol. 161, no. 2, pp. 185188, 1998.

[14] E. Sarti, P. M. Schantz, A. Plancarte et al., "Epidemiological investigation of Taenia solium taeniasis and cysticercosis in a rural village of Michoacan State, Mexico," Transactions of the Royal Society of Tropical Medicine and Hygiene, vol. 88, no. 1, pp. 49-52, 1994.

[15] R. J. Bobes, M. Hernández, C. Márquez et al., "Subarachnoidal and intraventricular human neurocysticercosis: application of an antigen detection assay for the diagnosis and follow-up," Tropical Medicine and International Health, vol. 11, no. 6, pp. 943-950, 2006.

[16] E. C. Bueno, C. M. Scheel, A. J. Vaz et al., "Application of synthetic 8-kD and recombinant GP50 antigens in the diagnosis of neurocysticercosis by enzyme-linked immunosorbent assay," American Journal of Tropical Medicine and Hygiene, vol. 72, no. 3, pp. 278-283, 2005.

[17] J. Y. Chung, Y. Y. Bahk, S. Huh, S. Y. Kong, Y. Kong, and S. Y. Cho, "A recombinant $10-\mathrm{kDa}$ protein of Taenia solium metacestodes specific to active neurocysticercosis," Journal of Infectious Diseases, vol. 180, no. 4, pp. 1307-1315, 1999.

[18] E. Ferrer, P. Bonay, M. Foster-Cuevas et al., "Molecular cloning and characterisation of Ts8B1, Ts8B2 and Ts8B3, three new members of the Taenia solium metacestode $8 \mathrm{kDa}$ diagnostic antigen family," Molecular and Biochemical Parasitology, vol. 152, no. 1, pp. 90-100, 2007.

[19] R. M. Greene, K. Hancock, P. P. Wilkins, and V. C. W. Tsang, "Taenia solium: molecular cloning and serologic evaluation of 14- and 18-kDa related, diagnostic antigens," Journal of Parasitology, vol. 86, no. 5, pp. 1001-1007, 2000.

[20] K. Hancock, A. Khan, F. B. Williams et al., "Characterization of the 8-kilodalton antigens of Taenia solium metacestodes and evaluation of their use in an enzyme-linked immunosorbent assay for serodiagnosis," Journal of Clinical Microbiology, vol. 41, no. 6, pp. 2577-2586, 2003.

[21] K. Hancock, S. Pattabhi, R. M. Greene et al., "Characterization and cloning of GP50, a Taenia solium antigen diagnostic for cysticercosis," Molecular and Biochemical Parasitology, vol. 133, no. 1, pp. 115-124, 2004.

[22] K. Hancock, S. Pattabhi, F. W. Whitfield et al., "Characterization and cloning of T24, a Taenia solium antigen diagnostic 
for cysticercosis," Molecular and Biochemical Parasitology, vol. 147, no. 1, pp. 109-117, 2006.

[23] R. C. R. Hell, P. Amim, H. M. de Andrade et al., "Immunodiagnosis of human neurocysticercosis using a synthetic peptide selected by phage-display," Clinical Immunology, vol. 131, no. 1, pp. 129-138, 2009.

[24] M. Hernández, C. Beltrán, E. García et al., "Cysticercosis: towards the design of a diagnostic kit based on synthetic peptides," Immunology Letters, vol. 71, no. 1, pp. 13-17, 2000.

[25] E. G. Lee, M. Y. Lee, J. Y. Chung et al., "Feasibility of baculovirus-expressed recombinant $10-\mathrm{kDa}$ antigen in the serodiagnosis of Taenia solium neurocysticercosis," Transactions of the Royal Society of Tropical Medicine and Hygiene, vol. 99, no. 12, pp. 919-926, 2005.

[26] M. O. Sato, Y. Sako, M. Nakao, H. Yamasaki, K. Nakaya, and A. Ito, "Evaluation of purified Taenia solium glycoproteins and recombinant antigens in the serologic detection of human and swine cysticercosis," Journal of Infectious Diseases, vol. 194, no. 12, pp. 1783-1790, 2006.

[27] M. R. M. da Silva, A. A. M. Maia, N. M. Espíndola, L. D. R. Machado, A. J. Vaz, and F. Henrique-Silva, "Recombinant expression of Taenia solium TS14 antigen and its utilization for immunodiagnosis of neurocysticercosis," Acta Tropica, vol. 100, no. 3, pp. 192-198, 2006.

[28] L. Salim, A. Ang, S. Handali, and V. C. W. Tsang, "Seroepidemiologic survey of cysticercosis-taeniasis in four central highland districts of papua, indonesia," American Journal of Tropical Medicine and Hygiene, vol. 80, no. 3, pp. 384-388, 2009.

[29] C. M. Scheel, A. Khan, K. Hancock et al., "Serodiagnosis of neurocysticercosis using synthetic 8-KD proteins: comparison of assay formats," American Journal of Tropical Medicine and Hygiene, vol. 73, no. 4, pp. 771-776, 2005.

[30] S. R. V. Atluri, P. Singhi, N. Khandelwal, and N. Malla, "Evaluation of excretory secretory and $10-30 \mathrm{kDa}$ antigens of Taenia solium cysticerci by EITB assay for the diagnosis of neurocysticercosis: brief definitive report," Parasite Immunology, vol. 31, no. 3, pp. 151-155, 2009.

[31] S. R. V. Atluri, P. Singhi, N. Khandelwal, and N. Malla, "Neurocysticercosis immunodiagnosis using Taenia solium cysticerci crude soluble extract, excretory secretory and lower molecular mass antigens in serum and urine samples of Indian children," Acta Tropica, vol. 110, no. 1, pp. 22-27, 2009.

[32] Y. Castillo, S. Rodriguez, H. H. García et al., "Urine antigen detection for the diagnosis of human neurocysticercosis," American Journal of Tropical Medicine and Hygiene, vol. 80, no. 3, pp. 379-383, 2009.

[33] N. Malla, R. Kaur, N. K. Ganguly, I. M. Sawhney, and R. C. Mahajan, "Utility of specific IgG4 response in saliva and serum samples for the diagnosis and follow up of human neurocysticercosis," Nepal Medical College Journal, vol. 7, no. 1, pp. 1-9, 2005.

[34] A. Flisser and T. W. Gyorkos, "Contribution of immunodiagnostic tests to epidemiological/intervention studies of cysticercosis/taeniosis in México," Parasite Immunology, vol. 29, no. 12, pp. 637-649, 2007.

[35] V. Prabhakaran, V. Rajshekhar, K. D. Murrell, and A. Oommen, "Conformation-sensitive immunoassays improve the serodiagnosis of solitary cysticercus granuloma in Indian patients," Transactions of the Royal Society of Tropical Medicine and Hygiene, vol. 101, no. 6, pp. 570-577, 2007.
[36] M. Ramos-Kuri, R. M. Montoya, A. Padilla et al., "Immunodiagnosis of neurocysticercosis: disappointing performance of serology (enzyme-linked immunosorbent assay) in an unbiased sample of neurological patients," Archives of Neurology, vol. 49, no. 6, pp. 633-636, 1992.

[37] S. J. Furrows, J. McCroddan, W. J. Bligh, and P. Chiodini, "Lack of specificity of a single positive $50-\mathrm{kDa}$ band in the electroimmunotransfer blot (EITB) assay for cysticercosis," Clinical Microbiology and Infection, vol. 12, no. 5, pp. 459462, 2006.

[38] A. Fleury, A. Dessein, P. M. Preux et al., "Symptomatic human neurocysticercosis: age, sex and exposure factors relating with disease heterogeneity," Journal of Neurology, vol. 251, no. 7, pp. 830-837, 2004.

[39] J. Morales-Montor, A. Chavarria, M. A. De León et al., "Host gender in parasitic infections of mammals: an evaluation of the female host supremacy paradigm," Journal of Parasitology, vol. 90, no. 3, pp. 531-546, 2004.

[40] J. Morales-Montor, S. Baig, C. Hallal-Calleros, and R. T. Damian, "Taenia crassiceps: androgen reconstitution of the host leads to protection during cysticercosis," Experimental Parasitology, vol. 100, no. 4, pp. 209-216, 2002.

[41] I. Marriott and Y. M. Huet-Hudson, "Sexual dimorphism in innate immune responses to infectious organisms," Immunologic Research, vol. 34, no. 3, pp. 177-192, 2006.

[42] A. Chavarría, A. Fleury, E. García, C. Márquez, G. Fragoso, and E. Sciutto, "Relationship between the clinical heterogeneity of neurocysticercosis and the immune-inflammatory profiles," Clinical Immunology, vol. 116, no. 3, pp. 271-278, 2005.

[43] O. H. Del Brutto, E. Garcia, O. Talamas, and J. Sotelo, "Sex-related severity of inflammation in parenchymal brain cysticercosis," Archives of Internal Medicine, vol. 148, no. 3, pp. 544-546, 1988.

[44] A. Fleury, T. Gomez, I. Alvarez et al., "High prevalence of calcified silent neurocysticercosis in a rural village of Mexico," Neuroepidemiology, vol. 22, no. 2, pp. 139-145, 2003.

[45] D. Verthelyi, "Sex hormones as immunomodulators in health and disease," International Immunopharmacology, vol. 1, no. 6, pp. 983-993, 2001.

[46] S. Khurana, A. Aggarwal, and N. Malla, "Prevalence of anticysticercus antibodies in slum, rural and urban populations in and around Union Territory, Chandigarh," Indian Journal of Pathology and Microbiology, vol. 49, no. 1, pp. 51-53, 2006.

[47] M. Tibayrenc, "Human genetic diversity and the epidemiology of parasitic and other transmissible diseases," Advances in Parasitology, vol. 64, pp. 377-462, 2007.

[48] C. Alves, T. Souza, I. Meyer, M. B. P. Toralles, and C. Brites, "Immunogenetics and infectious diseases: special reference to the mayor histocompatibility complex," Brazilian Journal of Infectious Diseases, vol. 10, no. 2, pp. 122-131, 2006.

[49] S. C. Shafir, F. J. Sorvillo, and L. Smith, "Current issues and considerations regarding trichomoniasis and human immunodeficiency virus in African-Americans," Clinical Microbiology Reviews, vol. 22, no. 1, pp. 37-45, 2009.

[50] F. Verra, P. Avellino, G. Bancone, V. Mangano, and D. Modiano, "Genetic epidemiology of susceptibility to malaria: not only academic exercises," Parassitologia, vol. 50, no. 1-2, pp. 147-150, 2008.

[51] O. H. Del Brutto, G. Granados, O. Talamas, J. Sotelo, and C. Gorodezky, "Genetic pattern of the HLA system: HLA A, B, C, DR, and DQ antigens in Mexican patients with parenchymal brain cysticercosis," Human Biology, vol. 63, no. 1, pp. 85-93, 1991. 
[52] G. Singh, "Neurocysticercosos in South-Central America and the Indian subcontinent. A comparative evaluation," Arquivos de Neuro-Psiquiatria, vol. 55, no. 3, pp. 349-356, 1997.

[53] R. Vega, D. Piñero, B. Ramanankandrasana et al., "Population genetic structure of Taenia solium from Madagascar and Mexico: Implications for clinical profile diversity and immunological technology," International Journal for Parasitology, vol. 33, no. 13, pp. 1479-1485, 2003.

[54] K. Hancock, D. E. Broughel, I. N. S. Moura et al., "Sequence variation in the cytochrome oxidase I, internal transcribed spacer 1, and Ts14 diagnostic antigen sequences of Taenia solium isolates from South and Central America, India, and Asia," International Journal for Parasitology, vol. 31, no. 14, pp. 1601-1607, 2001.

[55] M. Nakao, M. Okamoto, Y. Sako, H. Yamasaki, K. Nakaya, and A. Ito, "A phylogenetic hypothesis for the distribution of two genotypes of the pig tapeworm Taenia solium worldwide," Parasitology, vol. 124, no. 6, pp. 657-662, 2002.

[56] P. Maravilla, V. Souza, A. Valera et al., "Detection of Genetic Variation in Taenia solium," Journal of Parasitology, vol. 89, no. 6, pp. 1250-1254, 2003.

[57] G. Campbell, H. H. Garcia, M. Nakao, A. Ito, and P. S. Craig, "Genetic variation in Taenia solium," Parasitology International, vol. 55, supplement, pp. S121-S126, 2006.

[58] R. J. Bobes, G. Fragoso, M. D. R. Reyes-Montes et al., "Genetic diversity of Taenia solium cysticerci from naturally infected pigs of central Mexico," Veterinary Parasitology, vol. 168, no. 1-2, pp. 130-135, 2010.

[59] H. B. Oliveira, G. A. MacHado, M. D. R. F. GonçalvesPires, L. P. Moura, and J. M. Costa-Cruz, "Saline extract of Taenia saginata metacestodes as an alternative antigen for the immunodiagnosis of neurocysticercosis in human cerebrospinal fluid," Parasitology Research, vol. 105, no. 1, pp. 169-174, 2009.

[60] J. V. Proaño-Narvaez, A. Meza-Lucas, O. Mata-Ruiz, R. C. García-Jerónimo, and D. Correa, "Laboratory diagnosis of human neurocysticercosis: double-blind comparison of enzyme-linked immunosorbent assay and electroimmunotransfer blot assay," Journal of Clinical Microbiology, vol. 40, no. 6, pp. 2115-2118, 2002.

[61] H. H. Garcia, A. E. Gonzalez, R. H. Gilman et al., "Short report: transient antibody response in Taenia solium infection in field conditions-a major contributor to high seroprevalence," American Journal of Tropical Medicine and Hygiene, vol. 65, no. 1, pp. 31-32, 2001.

[62] P. Dekumyoy, J. Waikagul, S. Vanijanonta et al., "Cysticercosis: IgG-ELISA evaluations of peak1 antigen and $<30 \mathrm{kDa}$ antigen of delipidized extract of Taenia solium metacestodes," Southeast Asian Journal of Tropical Medicine and Public Health, vol. 35, no. 1, pp. 1-9, 2004.

[63] B. Gottstein, V. C. W. Tsang, and P. M. Schantz, "Demonstration of species-specific and cross-reactive components of Taenia solium metacestode antigens," American Journal of Tropical Medicine and Hygiene, vol. 35, no. 2, pp. 308-313, 1986.

[64] E. Hernandez-Cruz, J. J. González-Cabriales, C. OrdazPichardo, N. I. De La Cruz-Hernández, and G. H. FloresGutiérrez, "Development of an immunobinding dot-blot assay as an alternative for the serodiagnosis of human cysticercosis," Journal of Helminthology, vol. 83, no. 4, pp. 333-337, 2009.

[65] M. M. I. Ishida, G. Rubinsky-Elefant, A. W. Ferreira, S. Hoshino-Shimizu, and A. J. Vaz, "Helminth antigens (Taenia solium, Taenia crassiceps, Toxocara canis, Schistosoma mansoni and Echinococcus granulosus) and cross-reactivities in human infections and immunized animals," Acta Tropica, vol. 89, no. 1, pp. 73-84, 2003.

[66] N. M. Espíndola, A. H. Iha, I. Fernandes et al., "Cysticercosis immunodiagnosis using 18- and 14-kilodalton proteins from Taenia crassiceps cysticercus antigens obtained by immunoaffinity chromatography," Journal of Clinical Microbiology, vol. 43, no. 7, pp. 3178-3184, 2005.

[67] J. Mandal, P. D. Singhi, N. Khandelwal, and N. Malla, "Evaluation of lower molecular mass $(20-24 \mathrm{kDa})$ Taenia solium cysticercus antigen fraction by ELISA and dot blot for the serodiagnosis of neurocysticercosis in children," Parasitology Research, vol. 102, no. 5, pp. 1097-1101, 2008.

[68] G. C. Arruda, A. D. T. Da Silva, E. M. A. B. Quagliato, M. A. Maretti, and C. L. Rossi, "Evaluation of Taenia solium and Taenia crassiceps cysticercal antigens for the serodiagnosis of neurocysticercosis," Tropical Medicine and International Health, vol. 10, no. 10, pp. 1005-1012, 2005.

[69] S. Rodriguez, P. Dorny, V. C. W. Tsang et al., "Detection of Taenia solium antigens and anti-T. solium antibodies in paired serum and cerebrospinal fluid samples from patients with intraparenchymal or extraparenchymal neurocysticercosis," Journal of Infectious Diseases, vol. 199, no. 9, pp. 13451352, 2009.

[70] E. C. Bueno, A. J. Vaz, L. Machado, and J. A. Livramento, "Neurocysticercosis: detection of IgG, IgA and IgE antibodies in cerebrospinal fluid, serum and saliva samples by ELISA with Taenia solium and Taenia crassiceps antigens," Arquivos de Neuro-Psiquiatria, vol. 58, no. 1, pp. 18-24, 2000.

[71] H. H. Garcia, R. M. E. Parkhouse, R. H. Gilman et al., "Serum antigen detection in the diagnosis, treatment, and followup of neurocysticercosis patients," Transactions of the Royal Society of Tropical Medicine and Hygiene, vol. 94, no. 6, pp. 673-676, 2000.

[72] J. A. Lopez, E. Garcia, I. M. Cortes, J. Sotelo, P. Tato, and J. L. Molinari, "Neurocysticercosis: relationship between the developmental stage of metacestode present and the titre of specific IgG in the cerebrospinal fluid," Annals of Tropical Medicine and Parasitology, vol. 98, no. 6, pp. 569-579, 2004.

[73] J. L. Molinari, E. García-Mendoza, Y. De la Garza, J. A. Ramírez, J. Sotelo, and P. Tato, "Discrimination between active and inactive neurocysticercosis by metacestode excretory/secretory antigens of Taenia solium in an enzymelinked immunosorbent assay," American Journal of Tropical Medicine and Hygiene, vol. 66, no. 6, pp. 777-781, 2002.

[74] P. S. Sahu, S. C. Parija, S. K. Narayan, and D. Kumar, "Evaluation of an IgG-ELISA strategy using Taenia solium metacestode somatic and excretory-secretory antigens for diagnosis of neurocysticercosis revealing biological stage of the larvae," Acta Tropica, vol. 110, no. 1, pp. 38-45, 2009.

[75] J. S. Grewal, S. Kaur, G. Bhatti, N. K. Ganguly, R. C. Mahajan, and N. Malla, "Kinetics of humoral and cellular immune responses in experimental cysticercosis in pigs infected with Taenia solium," Indian Journal of Medical Research, vol. 111, pp. 43-49, 2000.

[76] J. P. Laclette, A. Alagon, K. Willms, and A. Torre-Blanco, "Purification of antigen B from Taenia solium cysticerci by affinity to mammalian collagen," Journal of Parasitology, vol. 76, no. 2, pp. 273-275, 1990.

[77] E. C. Bueno, M. Snege, A. J. Vaz, and P. G. Leser, "Serodiagnosis of human cysticercosis by using antigens from vesicular fluid of Taenia crassiceps cysticerci," Clinical and Diagnostic Laboratory Immunology, vol. 8, no. 6, pp. 1140-1144, 2001. 
[78] E. C. Bueno, A. J. Vaz, L. Machado, J. A. Livramento, and S. R. Mielle, "Specific Taenia crassiceps and Taenia solium antigenic peptides for neurocysticercosis immunodiagnosis using serum samples," Journal of Clinical Microbiology, vol. 38, no. 1, pp. 146-151, 2000.

[79] M. M. I. Ishida, R. H. S. Peralta, J. A. Livramento, S. HoshinoShimizu, J. M. Peralta, and A. J. Vaz, "Serodiagnosis of neurocysticercosis in patients with epileptic seizure using ELISA and immunoblot assay," Revista do Instituto de Medicina Tropical de Sao Paulo, vol. 48, no. 6, pp. 343-346, 2006.

[80] H. B. Oliveira, G. A. Machado, D. D. Cabral, and J. M. CostaCruz, "Application of Taenia saginata metacestodes as an alternative antigen for the serological diagnosis of human neurocysticercosis," Parasitology Research, vol. 101, no. 4, pp. 1007-1013, 2007.

[81] N. Shukla, N. Husain, Jyotsna, S. Gupta, and M. Husain, "Comparisons between scolex and membrane antigens of Cysticercus fasciolaris and Cysticercus cellulosae larvae for immunodiagnosis of neurocysticercosis," Journal of Microbiology, Immunology and Infection, vol. 41, no. 6, pp. 519-524, 2008.

[82] B. Espinoza, G. Ruiz-Palacios, and A. Tovar, "Characterization by enzyme-linked immunosorbent assay of the humoral immune response in patients with neurocysticercosis and its application in immunodiagnosis," Journal of Clinical Microbiology, vol. 24, no. 4, pp. 536-541, 1986.

[83] E. Garcia, G. Ordonez, and J. Sotelo, "Antigens from Taenia crassiceps cysticerci used in complement fixation, enzyme-linked immunosorbent assay, and Western blot (immunoblot) for diagnosis of neurocysticercosis," Journal of Clinical Microbiology, vol. 33, no. 12, pp. 3324-3325, 1995.

[84] V. C. W. Tsang, J. A. Brand, and A. E. Boyer, "An enzyme-linked immunoelectrotransfer blot assay and glycoprotein antigens for diagnosing human cysticercosis (Taenia solium)," Journal of Infectious Diseases, vol. 159, no. 1, pp. 5059, 1989.

[85] O. H. Del Brutto, R. Santibanez, C. A. Noboa, R. Aguirre, E. Diaz, and T. A. Alarcon, "Epilepsy due to neurocysticercosis: analysis of 203 patients," Neurology, vol. 42, no. 2, pp. 389392, 1992.

[86] V. Prabhakaran, V. Rajshekhar, K. D. Murrell, and A. Oommen, "Taenia solium metacestode glycoproteins as diagnostic antigens for solitary cysticercus granuloma in Indian patients," Transactions of the Royal Society of Tropical Medicine and Hygiene, vol. 98, no. 8, pp. 478-484, 2004.

[87] F. Aguilar-Rebolledo, A. Meza-Lucas, J. Torres et al., "Evaluation of the enzyme-linked immunoelectrotransfer blot assay for diagnosis of neurocysticercosis in children," Journal of Child Neurology, vol. 17, no. 6, pp. 416-420, 2002.

[88] E. Santamaría, A. Plancarte, and A. S. De Aluja, "The experimental infection of pigs with different numbers of Taenia solium eggs: immune response and efficiency of establishment," Journal of Parasitology, vol. 88, no. 1, pp. 6973, 2002.

[89] A. C. White and H. H. Garcia, "Recent developments in the epidemiology, diagnosis, treatment, and prevention of neurocysticercosis," Current Infectious Disease Reports, vol. 1, no. 5, pp. 434-440, 1999.

[90] J. Mandal, P. D. Singhi, N. Khandelwal, and N. Malla, "Evaluation of ELISA and dot blots for the serodiagnosis of neurocysticercosis, in children found to have single or multiple enhancing lesions in computerized tomographic scans of the brain," Annals of Tropical Medicine and Parasitology, vol. 100, no. 1, pp. 39-48, 2006.

[91] M. K. Katti and E. C. Bueno, "Are alternative sources of parasitic (cysticercal) antigens necessary for diagnosis of neurocysticercosis?" Journal of Clinical Microbiology, vol. 38, no. 9, pp. 3524-3525, 2000.

[92] M. Feldman, A. Plancarte, M. Sandoval, M. Wilson, and A. Flisser, "Comparison of two assays (EIA and EITB) and two samples (saliva and serum) for the diagnosis of neurocysticercosis," Transactions of the Royal Society of Tropical Medicine and Hygiene, vol. 84, no. 4, pp. 559-562, 1990.

[93] C. R. Almeida, E. P. Ojopi, C. M. Nunes et al., “Taenia solium DNA is present in the cerebrospinal fluid of neurocysticercosis patients and can be used for diagnosis," European Archives of Psychiatry and Clinical Neuroscience, vol. 256, no. 5, pp. 307-310, 2006.

[94] A. Plancarte, M. Fexas, and A. Flisser, "Reactivity in ELISA and dot blot of purified GP24, an immunodominant antigen of Taenia solium, for the diagnosis of human neurocysticercosis," International Journal for Parasitology, vol. 24, no. 5, pp. 733-738, 1994.

[95] E. Ferrer, L. M. González, J. Á. Martínez-Escribano et al., "Evaluation of recombinant HP6-Tsag, an $18 \mathrm{kDa}$ Taenia saginata oncospheral adhesion protein, for the diagnosis of cysticercosis," Parasitology Research, vol. 101, no. 3, pp. 517525, 2007.

[96] W. W. Gibbs, "Nanobodies," Scientific American, vol. 293, no. 2, pp. 78-83, 2005.

[97] N. Deckers, D. Saerens, K. Kanobana et al., "Nanobodies, a promising tool for species-specific diagnosis of Taenia solium cysticercosis," International Journal for Parasitology, vol. 39, no. 5, pp. 625-633, 2009.

[98] L. Ratier, M. Urrutia, G. Paris, L. Zarebski, A. C. Frasch, and F. A. Goldbaum, "Relevance of the diversity among members of the Trypanosoma cruzi trans-sialidase family analyzed with camelids single-domain antibodies," PLoS ONE, vol. 3, no. 10, Article ID e3524, 2008.

[99] K. A. Henderson, V. A. Streltsov, A. M. Coley et al., "Structure of an IgNAR-AMA1 complex: targeting a conserved hydrophobic cleft broadens malarial strain recognition," Structure, vol. 15, no. 11, pp. 1452-1466, 2007.

[100] J. Wesolowski, V. Alzogaray, J. Reyelt et al., "Single domain antibodies: promising experimental and therapeutic tools in infection and immunity," Medical Microbiology and Immunology, vol. 198, no. 3, pp. 157-174, 2009.

[101] T. Corona, D. Pascoe, and D. Gonzalez-Barranco, "Anticysticercous antibodies in serum and cerebrospinal fluid in patients with cerebral cysticercosis," Journal of Neurology Neurosurgery and Psychiatry, vol. 49, no. 9, pp. 1044-1049, 1986.

[102] I. N. Mohammad, D. C. Heiner, B. L. Miller, M. A. Goldberg, and I. G. Kagan, "Enzyme-linked immunosorbent assay for the diagnosis of cerebral cysticercosis," Journal of Clinical Microbiology, vol. 20, no. 4, pp. 775-779, 1984.

[103] N. Rosas, J. Sotelo, and D. Nieto, "ELISA in the diagnosis of neurocysticercosis," Archives of Neurology, vol. 43, no. 4, pp. 353-356, 1986.

[104] D. Zini, V. J.R. Farrell, and A. A. Wadee, "The relationship of antibody levels to the clinical spectrum of human neurocysticercosis," Journal of Neurology Neurosurgery and Psychiatry, vol. 53, no. 8, pp. 656-661, 1990.

[105] E. G. Lee, Y. A. Bae, S. H. Kim, S. P. Díaz-Camacho, Y. Nawa, and Y. Kong, "Serodiagnostic reliability of single-step 
enriched low-molecular weight proteins of Taenia solium metacestode of American and Asian isolates," Transactions of the Royal Society of Tropical Medicine and Hygiene, vol. 104, no. 10, pp. 676-683, 2010.

[106] S. Handali, M. Klarman, A. N. Gaspard et al., "Multiantigen print immunoassay for comparison of diagnostic antigens for Taenia solium cysticercosis and taeniasis," Clinical and Vaccine Immunology, vol. 17, no. 1, pp. 68-72, 2010.

[107] T. Schmitz, U. Felderhoff-Mueser, M. Sifringer, F. Groenendaal, S. Kampmann, and A. Heep, "Expression of soluble Fas in the cerebrospinal fluid of preterm infants with posthemorrhagic hydrocephalus and cystic white matter damage," Journal of Perinatal Medicine, vol. 39, no. 1, pp. 83$88,2011$.

[108] R. L. Hayes, G. Robinson, U. Muller, and K. K. Wang, "Translation of neurological biomarkers to clinically relevant platforms," Methods in Molecular Biology, vol. 566, pp. 303$313,2009$. 

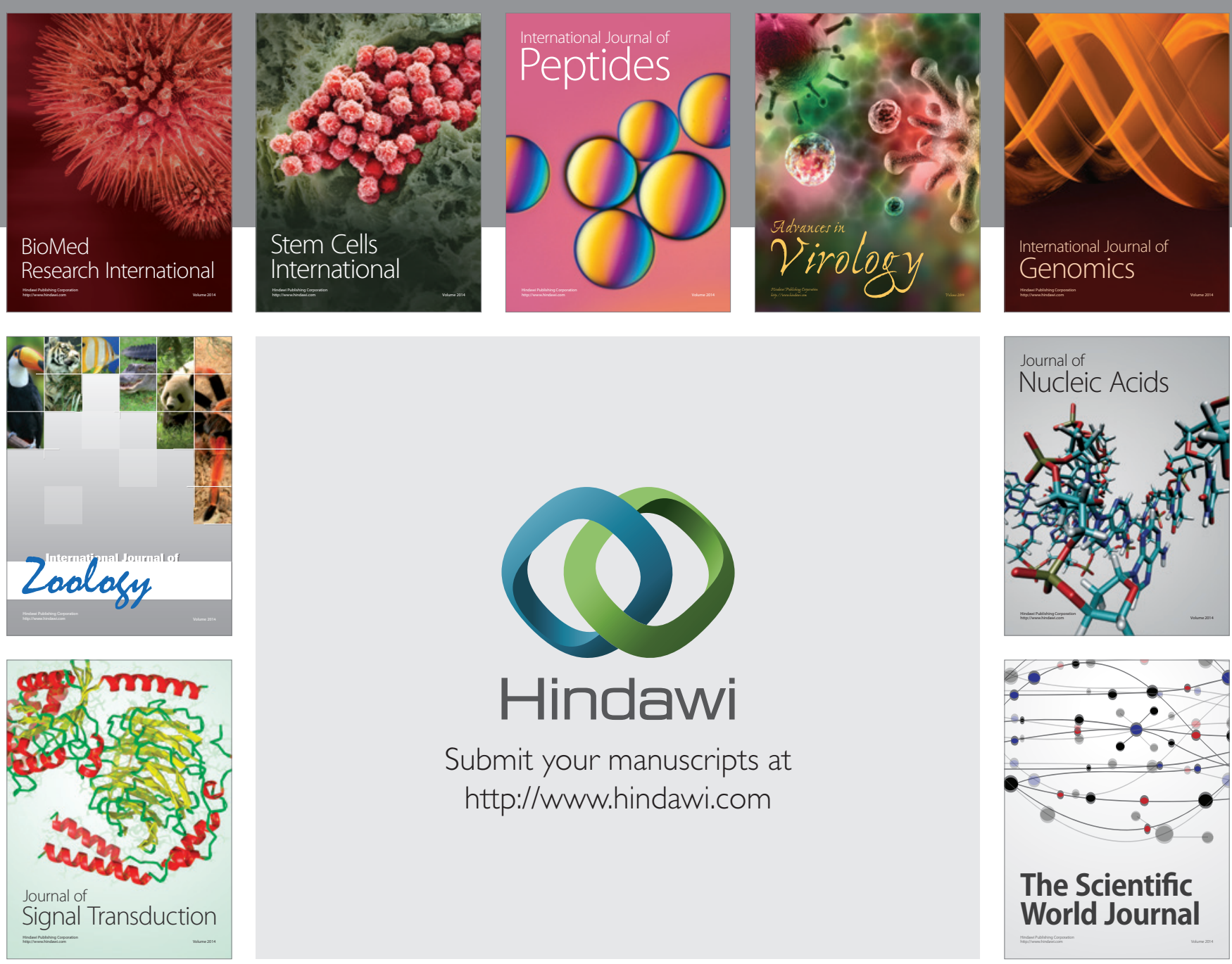

Submit your manuscripts at

http://www.hindawi.com
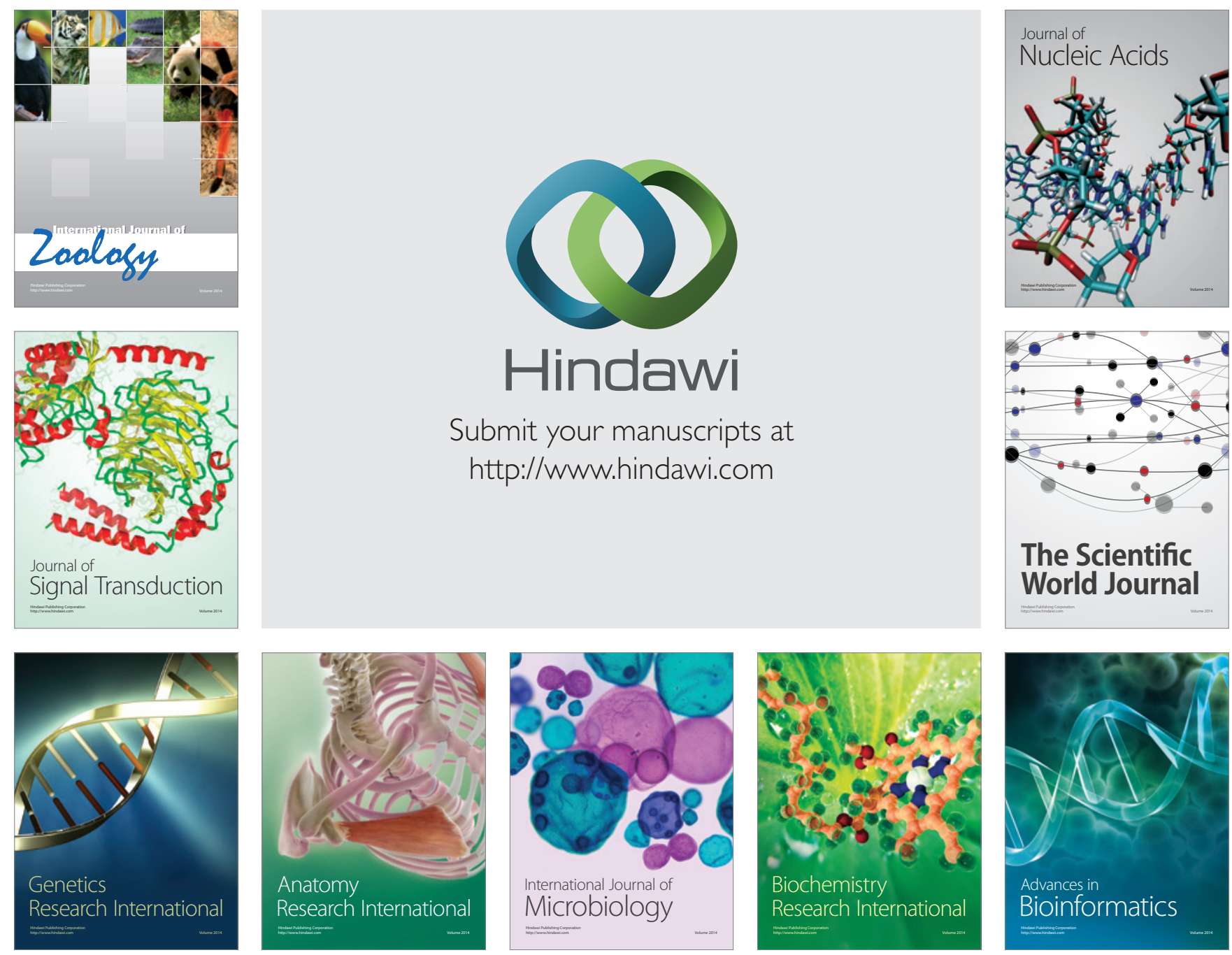

The Scientific World Journal
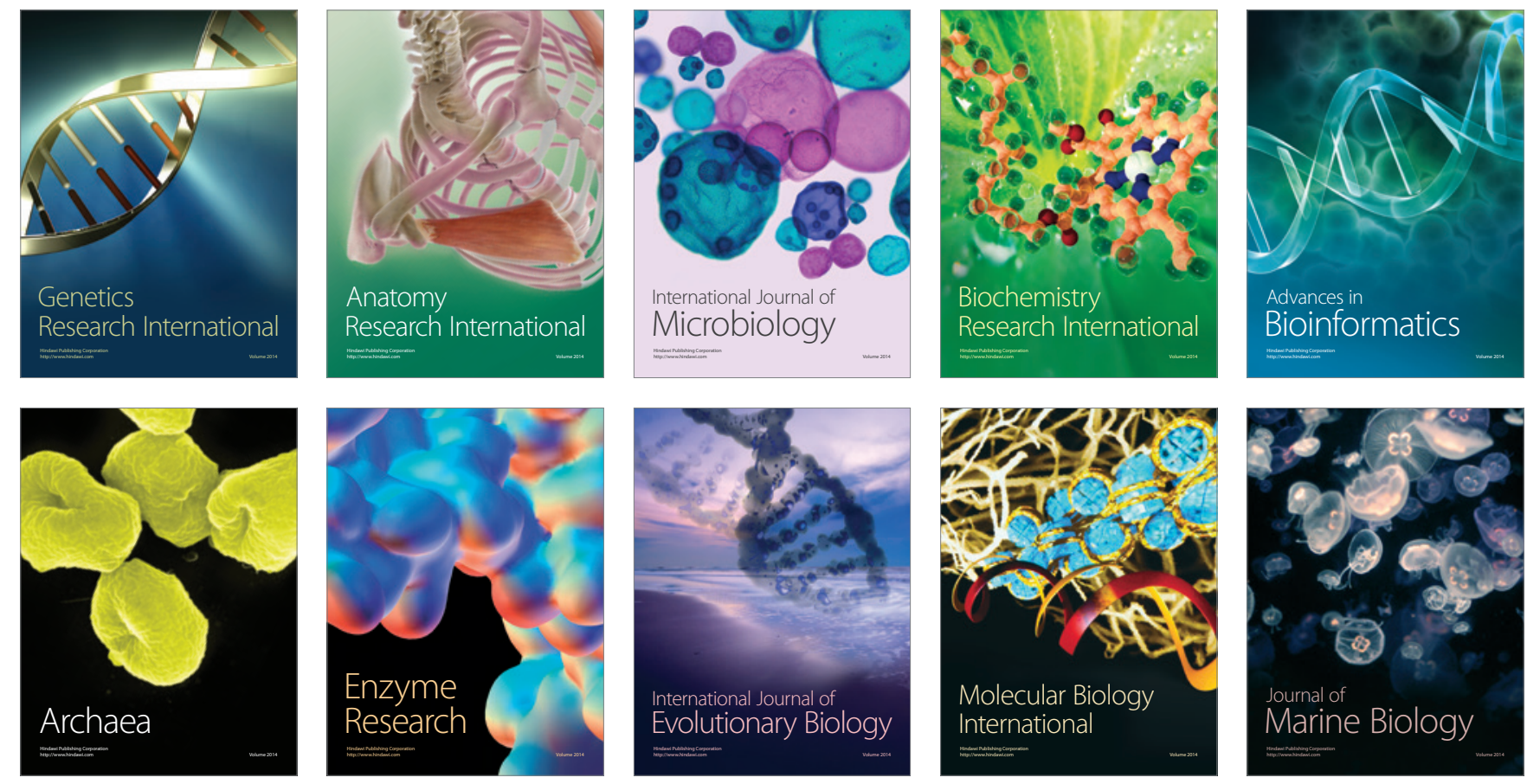